\title{
The Role of Polymorphisms in Vitamin D-Related Genes in Response to Vitamin D Supplementation
}

\author{
Sara Tomei ${ }^{1, *(\mathbb{D}}$, Parul Singh ${ }^{1}$, Rebecca Mathew ${ }^{1}$, Valentina Mattei ${ }^{1}$, Mathieu Garand ${ }^{1}(\mathbb{D}$, \\ Mariam Alwakeel $^{2} \mathbb{D}$, Elham Sharif $2, *,+\mathbb{D}$ and Souhaila Al Khodor ${ }^{1, *,+}$ \\ 1 Research Department, Sidra Medicine, Doha 26999, Qatar; psingh@sidra.org (P.S.); \\ rmathew1@sidra.org (R.M.); valentinamattei3@gmail.com (V.M.); mathieu.garand@gmail.com (M.G.) \\ 2 Department of Biomedical Sciences, College of Health Sciences, Qatar University, Doha 26999, Qatar; \\ mariamalwakee194@gmail.com \\ * Correspondence: stomei@sidra.org (S.T.); e.sharif@qu.edu.qa (E.S.); salkhodor@sidra.org (S.K.) \\ + Equal Senior authors.
}

Received: 16 July 2020; Accepted: 1 August 2020; Published: 27 August 2020

\begin{abstract}
Background. Vitamin D deficiency represents a major healthcare problem. Vitamin D status is influenced by genetic and environmental determinants. Several observational studies have evaluated the association of single-nucleotide polymorphisms (SNPs) in vitamin D-related genes and vitamin D levels. Nevertheless, little is known about the role of these SNPs in the response to vitamin D supplementation. We conducted an interventional study to define the association between SNPs in vitamin D-related genes and the response to vitamin D supplementation in 100 self-reported healthy women of Arab ancestry for the majority. Methods. A total of 100 healthy female subjects received a weekly oral dose of 50,000 IU vitamin D for 12 weeks. Serum vitamin D concentration and metabolic profiles were measured at baseline and 12 weeks post-vitamin D supplementation. The genotypes of 37 SNPs selected from previously reported vitamin D-related genes have been assessed by Fluidigm genotyping assay. Results. Rs731236 (VDR gene) and rs7116978 (CYP2R1 gene) showed a significant association with vitamin D status. The rs731236 GG genotype and the rs7116978 CC genotype were associated with a "vitamin D sufficiency" state. Rs731236 GG and rs7116978 CC genotypes showed a higher response to vitamin D supplementation. Transcription factor binding site prediction analysis showed altered binding sites for transcription factors according to the different rs7116978 alleles. Interestingly, the 37 SNPs previously established to play a role in vitamin D-related pathways explained very little of the response to vitamin D supplementation in our cohort, suggesting the existence of alternative loci whose number and effect size need to be investigated in future studies. Conclusion. In this paper, we present novel data on vitamin D-related SNPs and response to vitamin D supplementation demonstrating the feasibility of applying functional genomic approaches in interventional studies to assess individual-level responses to vitamin D supplementation.
\end{abstract}

Keywords: vitamin D; polymorphisms; single-nucleotide polymorphism; SNP; 25-hydroxyvitamin D; 25(OH)D; vitamin D deficiency; blood

\section{Introduction}

Vitamin D plays an important role in the endocrine system, and it takes part in several biological processes such as blood pressure regulation, calcium and phosphate homeostasis, nerve conduction, skeletal development, erythropoiesis, and so forth. [1-3]. The active form of vitamin D, 1,25-dihydroxyvitamin D [1,25(OH)2D], regulates the expression of vitamin D-related genes involved in calcium transport and bone matrix protein [4,5]. Nevertheless, vitamin D deficiency has been well documented worldwide [6]. Several factors have been shown to contribute to vitamin D 
deficiency, including low skin exposure to sunlight, low dietary intake of vitamin $\mathrm{D}$, high body mass index (BMI), genetic predisposition, the gut microbiome, and the immune system [7-11]. The Middle East regions are also affected by vitamin D deficiency [3,12-14]. In fact, despite having ample sunshine, these regions register the highest rate of vitamin D deficiency [13]. This is partially explained by the limited sun exposure due to cultural practices. Other common risk factors in these regions include female gender and clothing style, multiparity, sedentary lifestyle, and low intake of vitamin D and calcium from the diet $[14,15]$.

There are two forms of vitamin D: vitamin D2 and vitamin D3. Vitamin D2 mainly comes from fortified foods like breakfast cereals, milk, and other dairy items, while vitamin D3 is made by the body on exposure to sunlight [16]. In the bloodstream, vitamin D2 and vitamin D3 are converted to the major circulating form of vitamin D, which is 25-hydroxyvitamin D (25(OH)D) [17]. Serum 25(OH)D level is thus considered the best indicator of vitamin D supply to the body from both cutaneous synthesis and nutritional intake. Ethnic differences in the prevalence of common genetic polymorphisms provide an additional explanation for low vitamin D levels. Studies related to the role of the genetic background on the responsiveness to vitamin D supplementation are yet in their infancy $[18,19]$.

Noteworthy, while current studies provide data about vitamin D deficiency, they have been mainly focused on the Western populations [20-22], thus their conclusions do not necessarily apply to populations with a different genetic background. Additional research is warranted to understand the role of the genetic background in responsiveness to vitamin D supplementation, especially in regions disproportionally affected by vitamin D deficiency such as the Arab populations.

To date, observational and functional studies have been performed on vitamin D in Middle Eastern countries, however, interventional studies are currently lacking. The evidence from observational studies in humans is often susceptible to bias and confounders, thus provides limited evidence for causality. We designed this interventional study to test the hypothesis whether genetic polymorphisms in genes involved in the effect and/or metabolism of vitamin D3 influence the outcome of vitamin D3 supplementation by recruiting participants with low vitamin D levels (deficient or insufficient). We believe this question to be particularly relevant since little is known about the role of the genetic background in vitamin D deficiency and/or responsiveness to supplementation in the Arab population.

When selecting SNPs (single-nucleotide polymorphisms), we aimed at choosing the ones previously reported to be associated with vitamin D-related traits in addition to a few SNPs we wanted to explore [5]. These SNPs were selected from genes associated with vitamin D and included cytochrome P450 family 2, R (CYP2R1) [5,22-27], cytochrome P450 family 24 subfamily A member 1 (CYP24A1) [22,27-31], the 1-alpha-hydroxylase (CYP27B1) [32-35], the 7-dehydrocholesterol reductase/NAD synthetase 1 (DHCR7/NADSYN1) [26,36], the vitamin D receptor (VDR) [37,38], and the vitamin D-binding protein GC (group-specific component) [20-22,26,28,39,40].

\section{Materials and Methods}

\subsection{Study Subjects}

The study was approved by Qatar University (QU) Institutional Review Board (IRB) (QU-IRB; 531-A/15) and by Sidra Medicine IRB (1705010938). A total of 100 female students from QU were enrolled in the study. All subjects enrolled were self-reported as healthy and did not have any underlying diseases. Subjects were excluded if they were taking vitamin D or antibiotics, or were suffering from any chronic disease. Subjects were excluded from the final analysis if they failed to provide the blood sample at any stage of the study (pre- or post-supplementation).

An amount of $14 \mathrm{~mL}$ of peripheral blood was collected from each participating subject in phase 1 before vitamin D3 supplementation, and $6 \mathrm{~mL}$ of peripheral blood in phase 2 after vitamin D3 supplementation. In both phases, $4 \mathrm{~mL}$ of blood was used for biochemical assessment. The remaining $10 \mathrm{~mL}$ was used for molecular analyses. 
To classify the vitamin D levels S/D/I (sufficient, deficient, insufficient), pre- and postsupplementation, we followed the Endocrine Society criteria, where vitamin D deficiency is defined as serum $25(\mathrm{OH}) \mathrm{D}$ levels below $20 \mathrm{ng} / \mathrm{mL}$ (50 nmol/liter), vitamin $\mathrm{D}$ insufficiency is defined as $25(\mathrm{OH}) \mathrm{D}$ levels between 21 and $29 \mathrm{ng} / \mathrm{mL}$ (52.5-72.5 nmol/liter), and sufficiency is defined as serum $25(\mathrm{OH}) \mathrm{D}$ levels $\geq 30 \mathrm{ng} / \mathrm{mL}$ [41]. At the end of the intervention, participants were classified as either responder $(\mathrm{R})$ to vitamin $\mathrm{D}$ supplementation (those who achieved serum levels of $25(\mathrm{OH}) \mathrm{D}$ above $20 \mathrm{ng} / \mathrm{mL}$ ) or nonresponder (NR) (those whose serum levels of $25(\mathrm{OH})$ D remained $<20 \mathrm{ng} / \mathrm{mL}$ ) [19,42]. The biochemical measurements were performed at the Biomedical Labs in QU, while the molecular profiling was performed at Sidra Medicine.

\subsection{Anthropometric Measures}

The height was measured to the nearest $0.1 \mathrm{~cm}$ without shoes, using a Seca $\mathrm{GmbH} \& \mathrm{Co} . \mathrm{kg}$ stadiometer. WC (waist circumference, $\mathrm{cm}$ ) was measured midway between the lowest rib and the superior border of the iliac crest, just above the navel on standing subjects using an inelastic tape. Weight to the nearest $0.1 \mathrm{~kg}$ was measured in light clothing without shoes using a Seca GmbH \& Co. $\mathrm{kg}$ weight scale. BMI (Body Mass Index) was calculated as weight $(\mathrm{kg})$ divided by the squared height $\left(\mathrm{m}^{2}\right)$.

\subsection{Genetic Analysis of Polymorphisms Related to Vitamin D}

Blood samples were collected in tubes containing ethylenediaminetetraacetic acid EDTA as anticoagulant. Genomic DNA was isolated from peripheral blood using QIASymphony DSP DNA Midi kit (Qiagen, Hilden, Germany) following the manufacturer's recommendations. DNA quality and quantity were checked on Nanodrop 1000 (ThermoScientific, Waltham, MA, USA). The extracted DNA was used to amplify sequences containing polymorphisms related to vitamin D. These SNPs included: rs10783219, rs7139166, rs731236, rs757343, and rs4516035 in VDR gene; rs10741657, rs10766197, rs12794714, rs1562902, rs10500804, rs1993116, rs7116978, rs10877012, rs4646536, and rs703842 in CYP2R1 gene; rs11234027, rs12785878, and rs3829251 in DNCR7/NADSYN1 gene; rs1155563, rs12512631, rs16846876, rs17467825, rs222020, rs2282679, rs2298849, rs2298850, rs2882679, rs3755967, rs4588, and rs7041 in GC gene; rs17219315, rs2244719, rs229624, rs2296241, rs2426496, rs4809960, and rs6013897 in CYP24A1 gene.

SNP-specific assays were designed and ordered through the Fluidigm D3 Assay Design tool (https://d3.fluidigm.com/account/login). SNP-related sequences are reported in Supplementary Table S1. The genotypes of the polymorphisms listed above were determined by polymerase chain reaction (PCR). In the first step, two preamplification primers (locus-specific primer (LSP) and specific target amplification (STA) primer) were used to amplify the target region containing the SNP. The DNA sequences of the SNPs of interest were preamplified simultaneously in one multiplex PCR, for each sample separately, on a Veriti Thermal Cycler (Applied Biosystems, Foster City, CA, USA), with the following conditions: hold at $95^{\circ} \mathrm{C}$ for $15 \mathrm{~min}, 14$ cycles at $95^{\circ} \mathrm{C}$ for $15 \mathrm{~s}$, and $60^{\circ} \mathrm{C}$ for $4 \mathrm{~min}$. A second amplification was performed on the Fluidigm 96.96 Dynamic Array.

Assay mixture was prepared by mixing $3 \mu \mathrm{L}$ of each allele-specific primer (ASP), $8 \mu \mathrm{L}$ of each locus-specific primer (LSP), and $29 \mu \mathrm{L}$ DNA hydration buffer. One $\mu \mathrm{l}$ of each assay mix was combined with $2.5 \mu \mathrm{L}$ of $2 \times$ Assay Loading Reagent and $1.5 \mu \mathrm{L}$ of nuclease-free water. Sample mixture was prepared by combining $2.5 \mu \mathrm{L}$ of 1:50 diluted samples from the STA reaction and $3 \mu \mathrm{L}$ Biotium Fast Probe Master Mix. The BioMark HD dynamic array was first primed with control line fluid, and then loaded with the samples and assay mixtures via the appropriate inlets using an IFC (integrated fluidic circuit) controller. The array chip was placed in the BioMark HD Instrument. PCR was carried out using the following cycling conditions: $50^{\circ} \mathrm{C}$ for $2 \mathrm{~min}, 70^{\circ} \mathrm{C}$ for $30 \mathrm{~min}, 25^{\circ} \mathrm{C}$ for $10 \mathrm{~min}$, and $95^{\circ} \mathrm{C}$ for $5 \mathrm{~min}$, followed by four touchdown cycles $\left(95^{\circ} \mathrm{C}\right.$ for $15 \mathrm{~s}$, from $64^{\circ} \mathrm{C}$ to $61^{\circ} \mathrm{C}$ for $45 \mathrm{~s}, 72{ }^{\circ} \mathrm{C}$ for $15 \mathrm{~s}$ ) and 34 additional cycles $\left(95^{\circ} \mathrm{C}\right.$ for $15 \mathrm{~s}, 60^{\circ} \mathrm{C}$ for $45 \mathrm{~s}, 72{ }^{\circ} \mathrm{C}$ for $\left.15 \mathrm{~s}\right)$. Each PCR reaction used distilled water instead of DNA as negative control. Results were plotted on a two-dimensional scatter plot of the major versus the minor allele using the BioMark SNP Genotyping Analysis software version 2.1.1. 
Genotyping calls were assessed based on the allele discrimination plots and manually reviewed by looking at the single amplification plots. Genotyping calls were exported as a CSV file and processed for secondary analysis.

\subsection{Data Analysis}

Chi-square was used to test association and Hardy-Weinberg equilibrium (HWE) for each SNP. SNPs that did not pass the HWE criteria have been discarded from further analysis. All statistical assessments were two-sided and considered to be significant when $p$-value was $<0.05$. Pairwise comparisons and Bonferroni corrections were performed where relevant.

Genotyping data for the 37 SNPs under study were downloaded from the 1000 Genomes Project (http://www.internationalgenome.org) for a total of 5008 individuals (Current Build 152, released 2 October 2018, GRCh38.p12), including: Africans $(\mathrm{N}=1322)$, East Asians $(\mathrm{N}=1008)$, Europeans $(\mathrm{N}=1006)$, South Asians $(\mathrm{N}=978)$, and Americans $(\mathrm{N}=694)$. The Haploview 4.2 software package was used to estimate pairwise linkage disequilibrium (LD), and to detect departure from HWE, based on the expectation maximization algorithm [43]. Among all pairs of biallelic loci, we examined widely used measures of linkage disequilibrium (LD), Lewontin's D' ID'I and r2 [44]. All statistical tests were based on two-tailed probability. TFBS (transcription factor binding site) prediction analysis was also performed using PROMO software, version 8.3, ALGGEN.

SNP genotypes were categorized as either major, intermediate (Inter), or minor based on the frequency. The genotype category for each SNP of each donor was used to build a color grid (pheatmap, R: A Language and Environment for Statistical Computing) [45].

\section{Results}

All characteristics of the participants are shown in Table 1 . The mean age was 21 years. The mean BMI, height, waist circumference (WC), and weight were $24 \mathrm{~kg} / \mathrm{m}^{2}, 159 \mathrm{~cm}, 33$ inches, and $62 \mathrm{~kg}$, respectively. The participants had a mean level of ALT, AST, BUN, calcium, and creatinine in Phase 1 of $9.8 \mathrm{U} / \mathrm{L}, 15.1 \mathrm{U} / \mathrm{L}, 9.8 \mathrm{mg} / \mathrm{dl}, 9.2 \mathrm{mg} / \mathrm{dl}$, and $0.46 \mathrm{mg} / \mathrm{dL}$, respectively. The participants had a mean level of ALT, AST, BUN, calcium, and creatinine in Phase 2 of $12.9 \mathrm{U} / \mathrm{L}, 16.3 \mathrm{U} / \mathrm{L}, 12.7 \mathrm{mg} / \mathrm{dL}, 11.3 \mathrm{mg} / \mathrm{dl}$, and $0.67 \mathrm{mg} / \mathrm{dL}$, respectively. The mean level of the active form of vitamin $\mathrm{D}$ was 11.01 in Phase 1 and 34.3 in Phase 2. Table 1 also lists the 95\% confidence interval (CI) of the mean and the $p$-value associated with the hypothesis of null distribution.

Table 1. Characteristics of the participants $(\mathrm{N}=100)$.

\begin{tabular}{lccc}
\hline & Mean & $95 \%$ CI & Normal Distribution \\
\hline Age & 21.173 & $20.803-21.542$ & 0.0004 \\
\hline BMI (kg/m2) & 24.344 & $23.298-25.390$ & $<0.0001$ \\
\hline Height (cm) & 159.407 & $158.252-160.563$ & 0.7273 \\
\hline Phase 1 ALT (U/L) & 9.825 & $8.798-10.852$ & $<0.0001$ \\
\hline Phase 1 AST (U/L) & 15.072 & $13.967-16.176$ & 0.0015 \\
\hline Phase 1 BUN (mg/dl) & 9.821 & $9.195-10.447$ & 0.0109 \\
\hline Phase 1 Calcium (mg/dl) & 9.171 & $8.882-9.459$ & 0.6413 \\
\hline Phase 1 Creatinine (mg/dl) & 0.463 & $0.437-0.490$ & 0.4344 \\
\hline Phase 2 ALT (U/L) & 12.948 & $11.525-14.371$ & $<0.0001$ \\
\hline Phase 2 AST (U/L) & 16.344 & $15.118-17.571$ & 0.0016 \\
\hline Phase 2 BUN (mg/dl) & 12.664 & $11.883-13.446$ & 0.1309 \\
\hline
\end{tabular}


Table 1. Cont.

\begin{tabular}{lccc}
\hline & Mean & $\mathbf{9 5 \%}$ CI & Normal Distribution \\
\hline Phase 2 Calcium $(\mathrm{mg} / \mathrm{dl})$ & 11.324 & $10.996-11.652$ & 0.0367 \\
\hline Phase 2 Creatinine $(\mathrm{mg} / \mathrm{dl})$ & 0.674 & $0.639-0.708$ & 0.0009 \\
\hline Vitamin D Total Phase 1 & 11.014 & $9.989-12.038$ & 0.1375 \\
\hline Vitamin D Total Phase 2 & 34.332 & $31.430-37.234$ & 0.8644 \\
\hline Waist Circumference (inch) & 33.083 & $31.093-35.073$ & $<0.0001$ \\
\hline Weight $(\mathrm{kg})$ & 62.149 & $59.047-65.251$ & $<0.0001$ \\
\hline
\end{tabular}

The allele frequencies of the 37 SNPs assessed in this study are reported in Table 2. Four deviations from HWE were detected (SNP IDs: rs4516035, rs16846876, rs2298849, and rs2298850). The SNPs that did not meet HWE were removed from further analyses. One SNP (rs11234027) showed only one allele in the current cohort and was also removed from further analyses. We compared the allele distribution of the 37 SNPs with that of the populations included in the 1000 Genomes Project (1000G). Several SNPs showed differences in frequencies of the Ref (reference) allele in our cohort as compared to the data from the $1000 \mathrm{G}$ project. However, we cannot exclude that those differences are due to a small cohort size, or perhaps due to gender specificity.

Haplotype analysis was performed by chromosome (Chr.4 for GC gene, Chr. 11 for CYP2R1 and DHCR7 genes, Chr. 20 for CYP24A1 gene, Chr. 12 for CYP27B1 and VDR genes); no significant linkage disequilibrium was detected, probably due to the small cohort size (Supplementary Figure S1).

Table 2. Allele frequencies of the SNPs assessed in this study across the $1000 \mathrm{G}$ populations and the cohort from the current study. HWE: Hardy-Weinberg equilibrium; NA: not available. *, SNPs that did not pass HWE. All alleles in the table below are reported in the forward orientation.

\begin{tabular}{|c|c|c|c|c|c|c|c|c|c|c|}
\hline SNP ID & Gene & Chr. & Allele & $\begin{array}{l}\text { African } \\
(\mathrm{N}= \\
1322)\end{array}$ & $\begin{array}{c}\begin{array}{c}\text { East } \\
\text { Asian } \\
(\mathrm{N}= \\
1008)\end{array}\end{array}$ & $\begin{array}{c}\text { European } \\
(\mathrm{N}= \\
1006)\end{array}$ & $\begin{array}{l}\text { South Asian } \\
(\mathbf{N}=978)\end{array}$ & $\begin{array}{l}\text { American } \\
(\mathrm{N}=694)\end{array}$ & $\begin{array}{l}\text { Current } \\
\text { Study } \\
\text { Cohort } \\
(\mathrm{N}=89)\end{array}$ & $\begin{array}{c}\text { HWE } \\
p \text {-Value }\end{array}$ \\
\hline \multirow{2}{*}{ rs10783219 } & \multirow{2}{*}{ VDR } & \multirow{2}{*}{12} & $\operatorname{Ref}(\mathrm{T})$ & 0.023 & 0.441 & 0.332 & 0.33 & 0.51 & 0.55 & \multirow{2}{*}{0.29} \\
\hline & & & Alt (A) & 0.977 & 0.559 & 0.668 & 0.67 & 0.49 & 0.45 & \\
\hline \multirow{2}{*}{ rs7139166 } & \multirow{2}{*}{ VDR } & \multirow{2}{*}{12} & $\operatorname{Ref}(C)$ & 0.963 & 0.981 & 0.577 & 0.8 & 0.72 & 0.73 & \multirow{2}{*}{0.17} \\
\hline & & & Alt (G) & 0.037 & 0.019 & 0.423 & 0.2 & 0.28 & 0.27 & \\
\hline \multirow{2}{*}{ rs731236 } & \multirow{2}{*}{ VDR } & \multirow{2}{*}{12} & $\operatorname{Ref}(\mathrm{A})$ & 0.715 & 0.933 & 0.6 & 0.63 & 0.74 & 0.82 & \multirow{2}{*}{0.53} \\
\hline & & & Alt (G) & 0.285 & 0.067 & 0.4 & 0.37 & 0.26 & 0.18 & \\
\hline \multirow{2}{*}{ rs757343 } & \multirow{2}{*}{ VDR } & \multirow{2}{*}{12} & $\operatorname{Ref}(\mathrm{C})$ & 0.945 & 0.783 & 0.854 & 0.89 & 0.85 & 0.6 & \multirow{2}{*}{0.07} \\
\hline & & & Alt (T) & 0.055 & 0.217 & 0.146 & 0.11 & 0.15 & 0.4 & \\
\hline \multirow{2}{*}{ rs4516035 } & \multirow{2}{*}{ VDR } & \multirow{2}{*}{12} & $\operatorname{Ref}(\mathrm{T})$ & 0.963 & 0.981 & 0.578 & 0.8 & 0.72 & 0.6 & \multirow{2}{*}{$0.05^{*}$} \\
\hline & & & Alt (C) & 0.037 & 0.019 & 0.422 & 0.2 & 0.28 & 0.4 & \\
\hline \multirow{2}{*}{ rs10741657 } & \multirow{2}{*}{ CYP2R1 } & \multirow{2}{*}{11} & $\operatorname{Ref}(\mathrm{A})$ & 0.219 & 0.319 & 0.381 & 0.36 & 0.28 & 0.382 & \multirow{2}{*}{0.37} \\
\hline & & & Alt (G) & 0.781 & 0.681 & 0.619 & 0.64 & 0.72 & 0.618 & \\
\hline \multirow{2}{*}{ rs10766197 } & \multirow{2}{*}{ CYP2R1 } & \multirow{2}{*}{11} & $\operatorname{Ref}(\mathrm{G})$ & 0.896 & 0.643 & 0.526 & 0.55 & 0.64 & 0.15 & \multirow{2}{*}{0.97} \\
\hline & & & Alt (A) & 0.104 & 0.357 & 0.474 & 0.45 & 0.36 & 0.85 & \\
\hline \multirow{2}{*}{ rs12794714 } & \multirow{2}{*}{ CYP2R1 } & \multirow{2}{*}{11} & $\operatorname{Ref}(\mathrm{G})$ & 0.897 & 0.632 & 0.553 & 0.56 & 0.49 & 0.17 & \multirow{2}{*}{0.67} \\
\hline & & & Alt (A) & 0.103 & 0.368 & 0.447 & 0.44 & 0.51 & 0.83 & \\
\hline 1562002 & CYP2P1 & 11 & $\operatorname{Ref}(C)$ & 0.477 & 0.379 & 0.441 & 0.43 & 0.33 & 0.74 & 025 \\
\hline rs 1562902 & CYPZK1 & 11 & Alt (T) & 0.523 & 0.621 & 0.559 & 0.57 & 0.67 & 0.26 & 0.25 \\
\hline 10500804 & CYP2R1 & 11 & $\operatorname{Ref}(\mathrm{T})$ & 0.897 & 0.63 & 0.552 & 0.55 & 0.48 & 0.8 & 077 \\
\hline rS10500804 & CYPZKI & 11 & Alt (G) & 0.103 & 0.37 & 0.448 & 0.45 & 0.52 & 0.2 & 0.17 \\
\hline re1093116 & CYP2R1 & 11 & $\operatorname{Ref}(\mathrm{A})$ & 0.219 & 0.32 & 0.388 & 0.35 & 0.28 & 0.77 & 074 \\
\hline rS1993116 & CYPZK1 & 11 & Alt (G) & 0.781 & 0.68 & 0.612 & 0.65 & 0.72 & 0.23 & 0.74 \\
\hline
\end{tabular}


Table 2. Cont

\begin{tabular}{|c|c|c|c|c|c|c|c|c|c|c|}
\hline SNP ID & Gene & Chr. & Allele & $\begin{array}{l}\text { African } \\
(\mathrm{N}= \\
1322)\end{array}$ & $\begin{array}{c}\text { East } \\
\text { Asian } \\
(\mathrm{N}= \\
1008)\end{array}$ & $\begin{array}{l}\text { European } \\
(\mathrm{N}= \\
1006)\end{array}$ & $\begin{array}{l}\text { South Asian } \\
\text { ( } N=978)\end{array}$ & $\begin{array}{l}\text { American } \\
(\mathrm{N}=694)\end{array}$ & $\begin{array}{l}\text { Current } \\
\text { Study } \\
\text { Cohort } \\
(\mathrm{N}=89)\end{array}$ & $\begin{array}{c}\text { HWE } \\
p \text {-Value }\end{array}$ \\
\hline \multirow{2}{*}{ rs7116978 } & \multirow{2}{*}{ CYP2R1 } & \multirow{2}{*}{11} & $\operatorname{Ref}(\mathrm{T})$ & 0.329 & 0.32 & 0.344 & 0.34 & 0.28 & 0.46 & \multirow{2}{*}{0.37} \\
\hline & & & Alt (C) & 0.671 & 0.68 & 0.656 & 0.66 & 0.72 & 0.54 & \\
\hline \multirow{2}{*}{ rs10877012 } & \multirow{2}{*}{ CYP27B1 } & \multirow{2}{*}{12} & $\operatorname{Ref}(\mathrm{G})$ & 0.921 & 0.357 & 0.683 & 0.49 & 0.74 & 0.85 & \multirow{2}{*}{0.95} \\
\hline & & & Alt (T) & 0.079 & 0.643 & 0.317 & 0.51 & 0.26 & 0.15 & \\
\hline \multirow{2}{*}{ rs4646536 } & \multirow{2}{*}{ CYP27B1 } & \multirow{2}{*}{12} & $\operatorname{Ref}(\mathrm{A})$ & 0.756 & 0.354 & 0.681 & 0.42 & 0.72 & 0.03 & \multirow{2}{*}{0.37} \\
\hline & & & Alt (G) & 0.244 & 0.646 & 0.319 & 0.58 & 0.28 & 0.97 & \\
\hline \multirow{2}{*}{ rs703842 } & \multirow{2}{*}{ CYP27B1 } & \multirow{2}{*}{12} & $\operatorname{Ref}(\mathrm{A})$ & 0.7 & 0.354 & 0.68 & 0.42 & 0.71 & 0.15 & \multirow{2}{*}{0.1} \\
\hline & & & Alt (G) & 0.3 & 0.646 & 0.32 & 0.58 & 0.29 & 0.85 & \\
\hline \multirow{2}{*}{ rs11234027 } & 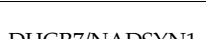 & 11 & $\operatorname{Ref}(\mathrm{G})$ & 0.651 & 0.696 & 0.808 & 0.65 & 0.76 & 0 & NIt \\
\hline & DHCR//NADSYNI & 11 & Alt (A) & 0.349 & 0.304 & 0.192 & 0.35 & 0.24 & 1 & NA \\
\hline rc12785878 & & & $\operatorname{Ref}(\mathrm{G})$ & 0.83 & 0.62 & 0.299 & 0.85 & 0.55 & 0.57 & \\
\hline rs12785878 & DHCR7/NADSYN1 & 11 & Alt (T) & 0.17 & 0.38 & 0.701 & 0.15 & 0.45 & 0.43 & 0.38 \\
\hline (1 & 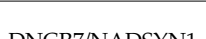 & 11 & $\operatorname{Ref}(\mathrm{G})$ & 0.743 & 0.702 & 0.809 & 0.65 & 0.77 & 0.15 & \\
\hline rs3829251 & DNCR//NADSYNI & 11 & Alt (A) & 0.257 & 0.298 & 0.191 & 0.35 & 0.23 & 0.85 & 0.94 \\
\hline 51155562 & $C C_{1}$ & 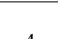 & $\operatorname{Ref}(\mathrm{T})$ & 0.943 & 0.664 & 0.756 & 0.68 & 0.81 & 0.6 & 010 \\
\hline rs1150563 & Ge & 4 & Alt (C) & 0.057 & 0.336 & 0.244 & 0.32 & 0.19 & 0.4 & 0.14 \\
\hline 12512621 & $C \Gamma$ & a & $\operatorname{Ref}(\mathrm{T})$ & 0.647 & 0.775 & 0.653 & 0.74 & 0.57 & 0.75 & 010 \\
\hline rs12512631 & $G C$ & 4 & Alt (C) & 0.353 & 0.225 & 0.347 & 0.26 & 0.43 & 0.25 & 0.19 \\
\hline 16016076 & $C G$ & e & $\operatorname{Ref}(\mathrm{A})$ & 0.853 & 0.74 & 0.702 & 0.7 & 0.78 & 0.403 & $004 *$ \\
\hline rs168468/6 & GC & 4 & Alt (T) & 0.147 & 0.26 & 0.298 & 0.3 & 0.22 & 0.596 & $0.04^{\star}$ \\
\hline $1716702-$ & $C C^{-}$ & e & $\operatorname{Ref}(\mathrm{A})$ & 0.946 & 0.739 & 0.751 & 0.7 & 0.79 & 0.72 & קרת \\
\hline rs $1746 / 825$ & GC & 4 & Alt (G) & 0.054 & 0.261 & 0.249 & 0.3 & 0.21 & 0.28 & 0.32 \\
\hline & & & $\operatorname{Ref}(\mathrm{C})$ & 0.633 & 0.416 & 0.162 & 0.13 & 0.21 & 0.73 & \\
\hline rs222020 & GC & 4 & Alt (T) & 0.367 & 0.584 & 0.838 & 0.87 & 0.79 & 0.27 & 0.17 \\
\hline 2020270 & $C C^{-1}$ & 等 & $\operatorname{Ref}(\mathrm{T})$ & 0.95 & 0.739 & 0.753 & 0.7 & 0.79 & 0.12 & \\
\hline rSZ28 $26 / 9$ & GC & 4 & Alt (G) & 0.05 & 0.261 & 0.247 & 0.3 & 0.21 & 0.88 & 0.79 \\
\hline (200890 & $C C_{1}$ & a & $\operatorname{Ref}(\mathrm{A})$ & 0.573 & 0.599 & 0.801 & 0.84 & 0.78 & 0.6 & $005 *$ \\
\hline rs2298849 & GC & 4 & Alt (G) & 0.427 & 0.401 & 0.199 & 0.16 & 0.22 & 0.4 & $0.05^{*}$ \\
\hline 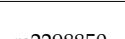 & $C C_{1}$ & t & $\operatorname{Ref}(\mathrm{G})$ & 0.974 & 0.74 & 0.764 & 0.71 & 0.8 & 0.75 & $002 *$ \\
\hline rs2298850 & $G C$ & 4 & Alt (C) & 0.026 & 0.26 & 0.236 & 0.29 & 0.2 & 0.25 & $0.02^{*}$ \\
\hline 202802670 & $C \Gamma$ & 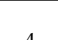 & $\operatorname{Ref}(\mathrm{C})$ & NA & NA & NA & NA & NA & 0.74 & 000 \\
\hline rs2882679 & GC & 4 & Alt (T) & NA & NA & NA & NA & NA & 0.26 & 0.99 \\
\hline 2755067 & $C C_{1}$ & 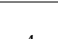 & $\operatorname{Ref}(C)$ & 0.946 & 0.739 & 0.752 & 0.7 & 0.79 & 0.19 & 020 \\
\hline rs3/55967 & $G C$ & 4 & Alt (T) & 0.054 & 0.261 & 0.248 & 0.3 & 0.21 & 0.81 & 0.38 \\
\hline tree & 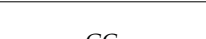 & & $\operatorname{Ref}(\mathrm{G})$ & 0.933 & 0.739 & 0.752 & 0.7 & 0.79 & 0.77 & \\
\hline rs4588 & GC & 4 & Alt (T) & 0.067 & 0.261 & 0.248 & 0.3 & 0.21 & 0.23 & 0.29 \\
\hline & & & $\operatorname{Ref}(\mathrm{A})$ & 0.906 & 0.7 & 0.417 & 0.46 & 0.46 & 0.75 & 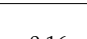 \\
\hline rs7041 & GC & 4 & Alt (C) & 0.094 & 0.3 & 0.583 & 0.54 & 0.54 & 0.25 & 0.16 \\
\hline $1721001-$ & CYDPA & 0 & $\operatorname{Ref}(\mathrm{A})$ & 0.997 & 1 & 0.982 & 0.98 & 0.99 & 0.22 & 0 \\
\hline $\mathrm{rs} 1 / 219315$ & CYP24AI & 20 & Alt (G) & 0.003 & 0 & 0.018 & 0.02 & 0.01 & 0.88 & 0.43 \\
\hline 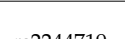 & CVDOA 1 & 0 & $\operatorname{Ref}(\mathrm{C})$ & 0.239 & 0.129 & 0.428 & 0.29 & 0.52 & 0.15 & 007 \\
\hline rs2244719 & CYP24AI & 20 & Alt (T) & 0.761 & 0.871 & 0.572 & 0.71 & 0.48 & 0.85 & 0.97 \\
\hline (2020624 & CYP2A 1 & 0 & $\operatorname{Ref}(\mathrm{G})$ & 0.893 & 0.969 & 0.815 & 0.8 & 0.9 & 0.53 & 025 \\
\hline rs 229624 & CYP24AI & 20 & Alt (A) & 0.107 & 0.031 & 0.185 & 0.2 & 0.1 & 0.47 & 0.35 \\
\hline (2) & C) & 0 & $\operatorname{Ref}(\mathrm{G})$ & 0.508 & 0.594 & 0.467 & 0.58 & 0.58 & 0.54 & 010 \\
\hline rs2296241 & CYP24A1 & 20 & Alt (A) & 0.492 & 0.406 & 0.533 & 0.42 & 0.42 & 0.46 & 0.19 \\
\hline & CYD24A 1 & & $\operatorname{Ref}(\mathrm{T})$ & 0.432 & 0.357 & 0.324 & 0.35 & 0.24 & 0.74 & 000 \\
\hline rs2426496 & CYP24A1 & 20 & Alt (G) & 0.568 & 0.643 & 0.676 & 0.65 & 0.76 & 0.26 & 0.99 \\
\hline & & & $\operatorname{Ref}(\mathrm{T})$ & 0.905 & 0.78 & 0.769 & 0.75 & 0.79 & 0.82 & 000 \\
\hline rs4809960 & CYP24A1 & 20 & Alt (C) & 0.095 & 0.22 & 0.231 & 0.25 & 0.21 & 0.18 & 0.84 \\
\hline 6017007 & CYDמ 1 & 0 & $\operatorname{Ref}(\mathrm{T})$ & 0.74 & 0.851 & 0.758 & 0.67 & 0.63 & 0.72 & 027 \\
\hline rS6013897 & CYP24AI & 20 & Alt (A) & 0.26 & 0.149 & 0.242 & 0.33 & 0.37 & 0.28 & 0.37 \\
\hline
\end{tabular}


We also assessed the association of each SNP with the response to vitamin D supplementation (i.e., R/NR; responder, nonresponder) and pretreatment vitamin D status (i.e., S/D/I; sufficient, deficient, insufficient) classification. Out of all the SNPs assessed, rs731236 (VDR gene) and rs7116978 (CYP2R1 gene) showed a significant association with S/D/I classification (two-tailed chi-square test, $p=0.0336$ and $p=0.0163$, respectively, shown in bold in Table 3 ). Figure 1 shows the percentage of the genotypes of the two SNPs mentioned above and according to the S/D/I classification. The rs731236 GG genotype and the rs7116978 CC genotype were associated with a "vitamin D sufficiency" state.

Table 3. Association of genotypes with R/NR and S/D/I (chi-square). Significant $p$-values are in bold.

\begin{tabular}{|c|c|c|c|}
\hline SNP ID & Gene & $p$-Value (R/NR) & $p$-Value (S/D/I) \\
\hline rs10783219 & VDR & 0.6071 & 0.5330 \\
\hline rs7139166 & VDR & 0.4249 & 0.6209 \\
\hline rs731236 & VDR & 0.5133 & 0.0336 \\
\hline rs757343 & VDR & 0.6023 & 0.6926 \\
\hline rs10741657 & CYP2R1 & 0.3748 & 0.4870 \\
\hline rs10766197 & CYP2R1 & 0.7235 & 0.3820 \\
\hline rs12794714 & CYP2R1 & 0.7221 & 0.8857 \\
\hline rs1562902 & CYP2R1 & 0.3136 & 0.1389 \\
\hline rs10500804 & CYP2R1 & 0.7443 & 0.8867 \\
\hline rs1993116 & CYP2R1 & 0.6900 & 0.6161 \\
\hline rs7116978 & CYP2R1 & 0.0727 & 0.0163 \\
\hline rs10877012 & CYP27B1 & 0.7108 & 0.3583 \\
\hline rs4646536 & CYP27B1 & 0.8004 & 0.8265 \\
\hline rs703842 & CYP27B1 & 0.7845 & 0.7230 \\
\hline rs12785878 & DHCR7/NADSYN1 & 0.9386 & 0.9820 \\
\hline rs3829251 & DHCR7/NADSYN1 & 0.7488 & 0.4279 \\
\hline rs1155563 & GC & 0.9104 & 0.9904 \\
\hline rs12512631 & GC & 0.3288 & 0.1126 \\
\hline rs17467825 & GC & 0.0661 & 0.1188 \\
\hline rs222020 & GC & 0.4039 & 0.4397 \\
\hline rs2282679 & GC & 0.9068 & 0.7540 \\
\hline rs2882679 & GC & 0.4395 & 0.6346 \\
\hline rs3755967 & GC & 0.8826 & 0.5654 \\
\hline rs4588 & GC & 0.2287 & 0.1922 \\
\hline rs7041 & GC & 0.1961 & 0.2328 \\
\hline rs17219315 & CYP24A1 & 0.6713 & 0.3635 \\
\hline rs2244719 & CYP24A1 & 0.7235 & 0.3820 \\
\hline rs229624 & CYP24A1 & 0.8586 & 0.8796 \\
\hline rs2296241 & CYP24A1 & 0.3863 & 0.4631 \\
\hline rs2426496 & CYP24A1 & 0.4395 & 0.6346 \\
\hline rs4809960 & CYP24A1 & 0.5668 & 0.1768 \\
\hline rs6013897 & CYP24A1 & 0.7249 & 0.9156 \\
\hline
\end{tabular}


Rs731236

A

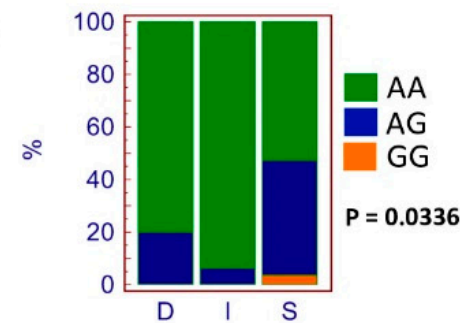

C

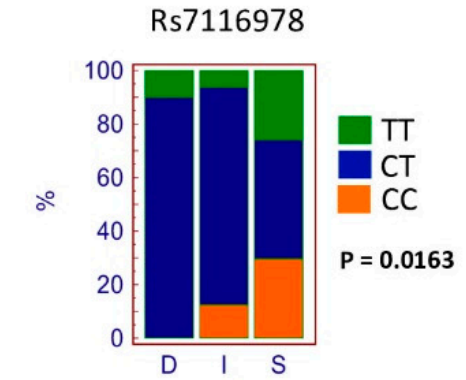

Rs731236 (VDR)

B

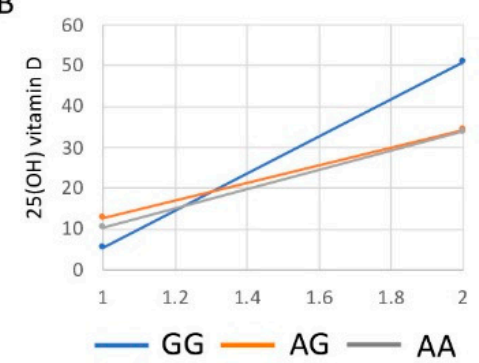

Rs7116978 (CYP2R1)

D

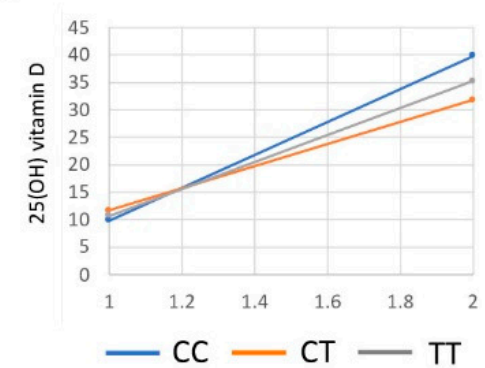

Figure 1. SNPs significantly associated with Deficient (D)/Insufficient (I)/Sufficient (S) classification. Genotype distribution according to Deficient (D)/Insufficient (I)/Sufficient (S) classification for rs731236 (A). Increment of vitamin D level from Phase 1 to Phase 2 according to rs731236 genotypes (B). Genotype distribution according to Deficient (D)/Insufficient (I)/Sufficient (S) classification for rs7116978 (C). Increment of vitamin D level from Phase 1 to Phase 2 according to rs7116978 genotypes (D).

To better understand the association of rs731236 and rs7116978 SNPs with the vitamin D sufficiency state, we performed pairwise comparisons by creating $3 \times 2$ contingency tables. For rs731236, only the contingency table of AA, AG, and GG genotypes versus I (Insufficient) and S (Sufficient) classification produced a significant $p$-value (chi-square $=8.868 ; p$-value $=0.0119$ ). For rs7116978, the two contingency tables of CC, CT, and TT genotypes versus D (Deficient) and S (Sufficient) classification and of CC, $\mathrm{CT}$, and TT genotypes versus I (Insufficient) and S (Sufficient) classification produced a significant $p$-value (CC,CT,TT vs. D,S: chi-square $=7.272, p$-value $=0.0264, \mathrm{CC}, \mathrm{CT}, \mathrm{TT}$ vs. I,S: chi-square $=6.802, p$-value $=0.0333$ ). Nevertheless, after applying Bonferroni correction, only the pairwise comparison rs731236 AA, AG, and GG genotypes versus I and S classification remained significant $(p$-value $=0.0357)$. We cannot exclude this to be due to the small cohort size.

The increase of serum $25(\mathrm{OH})$ vitamin D level (delta vitamin D values, difference between Phase 1 and Phase 2) is also shown according to the different genotypes. For SNP rs731236, genotype GG showed a higher increase as compared to AG and AA genotypes. As SNP rs7116978 is intronic, we have assessed whether the different alleles may alter the binding sites for transcription factors. The results of these analyses are reported in Supplementary Figure S2.

Figure 2 shows the allelic distribution of rs731236 and rs7116978 among the populations included in 1000G and the cohort included in the current study. For rs731236, the current study displayed a lower frequency of the $G$ allele as compared to the African, European, South Asian, and American cohorts. Allelic frequencies of rs7116978 were more similar across the populations included in 1000G. However, the current study showed a higher frequency of rs7116978 T allele as compared to all the other populations assessed. We cannot exclude the possibility that these differences are related to gender specificity; those differences may also not be represented by a small cohort as the one employed in this study. 
A

rs731236

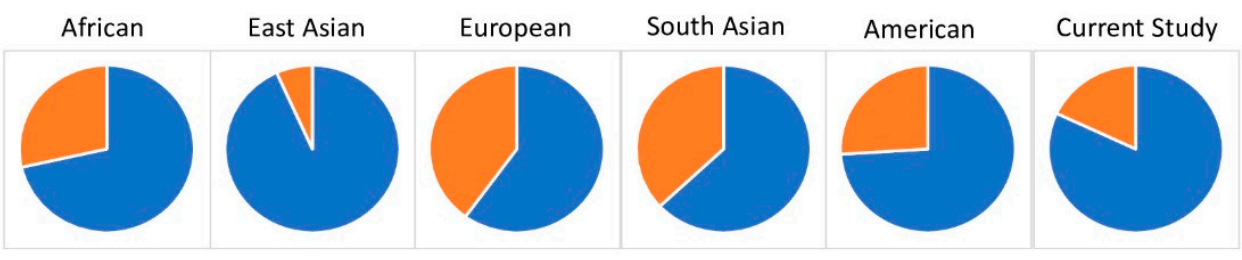

B $\quad$ rs7116978

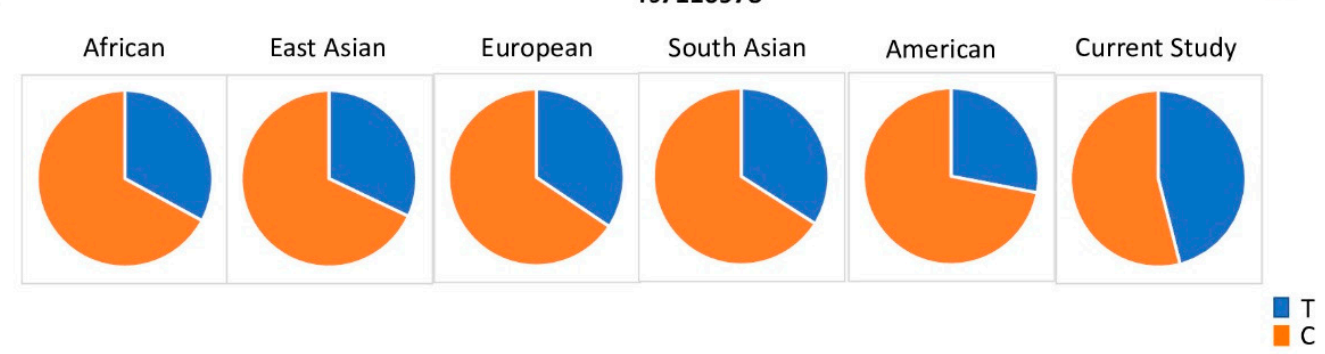

Figure 2. Distribution of rs731236 (A) and rs7116978 (B) allelic frequencies among world populations included in the 1000 Genomes Project and the cohort in our study.

We further sought to test whether the combination of different SNPs may predict R versus NR classification. Figure 3 shows heatmaps of SNPs categorized according to the genes they belong to. The heatmaps shown display the hierarchical clustering (complete-linkage clustering based on Euclidean distance) of SNPs and donors based on genotype. Horizontally, at the top of the heatmaps, we added color-coded labels that correspond to the genes. The hierarchical clustering of the donors (vertical hierarchical tree, left side of heatmaps) showed that groups formed based on the genotype of certain SNPs. No clear grouping of the participants based on their response to vitamin D supplementation was found in any of the genes assessed, suggesting that the genetic component alone does not predict responsiveness to vitamin $\mathrm{D}$ in our cohort. 

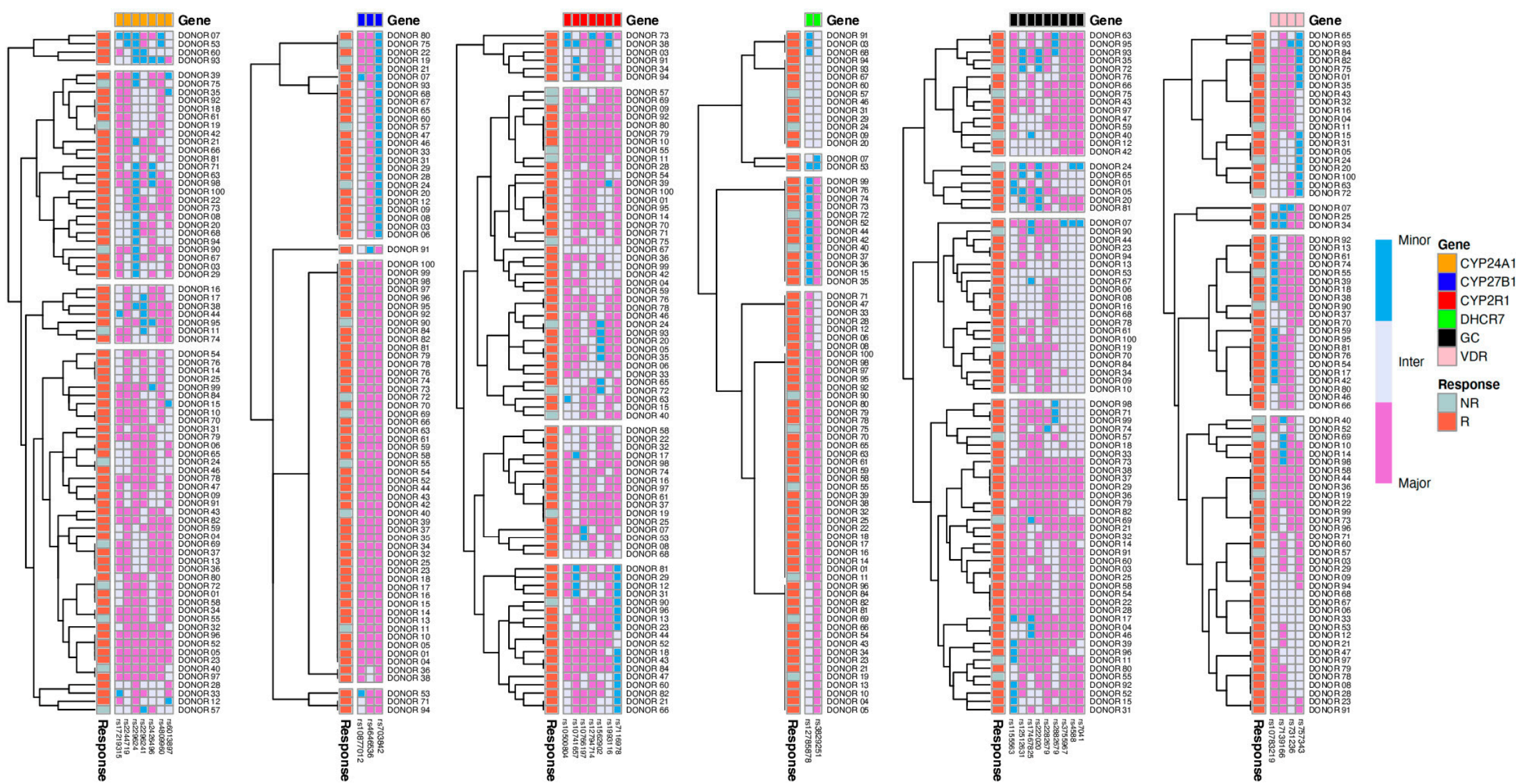

Figure 3. Hierarchical clustering and heatmaps based on genotyping results. Heatmaps display the hierarchical clustering (complete-linkage clustering based on Euclidean distance) of SNPs and donors based on genotype. Horizontally, at the top of the heatmaps, we added color-coded labels that correspond to the genes. The hierarchical clustering of the donors (vertical hierarchical tree, left side of heatmaps) showed that groups formed based on the genotype of certain SNPs. Genotypes are color-coded according to their frequencies, Major = pink, Inter = white, Minor = blue. SNPs that did not pass HWE were excluded from this picture. Labels of the $\mathrm{x}$-axis indicate R: responder and NR: nonresponder. 


\section{Discussion}

Vitamin D is a fat-soluble vitamin that is produced when the skin is exposed to UVB radiation [46]. Vitamin D deficiency is associated with chronic liver [47] and kidney [48] diseases.

The actions of vitamin D are mediated by several proteins. VDR (vitamin D receptor) is a ligand-activated transcription factor that acts through vitamin $\mathrm{D}$ response elements located near the start sites of target genes to regulate gene expression [49]. GC (group-specific component) encodes for a vitamin $\mathrm{D}$ binding protein that plays an important role in the transport and metabolism of vitamin $\mathrm{D}$, being the major plasma carrier for vitamin $\mathrm{D}$ and its metabolites [50]. The vitamin $\mathrm{D}$ pathway also involves a series of cytochrome P450-containing sterol hydroxylases that generate and degrade the active hormone serving as a ligand for the vitamin $\mathrm{D}$ receptor-mediated transcriptional gene expression [51]. Among these hydroxylases, CYP2R1 is the principal enzyme carrying the hydroxylation of vitamin D to 25-hydroxyvitamin D in the liver [52]; the 1a-hydroxylation of 25(OH)D in the kidney by CYP27B1 generates the fully active vitamin D metabolite [51,52]. Cytochrome P450 family 24 subfamily A member 1 (CYP24A1) gene encodes a mitochondrial monooxygenase which catalyzes the 24-hydroxylation of 1,25-dihydroxyvitamin D3 [51].

Recent studies have suggested that SNPs within the genes above may influence the level or activity of vitamin D, but studies exploring the association of SNPs in vitamin D-related genes with the response to supplementation of vitamin D are still lacking.

The present interventional study assessed the association between SNPs previously established to play a role in vitamin D-related genes and the responsiveness to vitamin D supplementation after the intervention. Our data showed that both rs731236 (VDR gene) and rs7116978 (CYP2R1 gene) have a significant association with the vitamin D status, where the rs731236 GG and the rs7116978 CC genotypes were associated with a "vitamin D sufficiency" state. In addition, rs731236 GG and rs7116978 CC genotypes were associated with a higher response to vitamin D supplementation. With the exception of rs731236 and rs7116978, the remaining SNPs previously established to play a role in vitamin D-related pathways explained very little the response to vitamin D supplementation in our cohort, suggesting the existence of alternative loci whose number and effect size warrant future studies.

Rs7116978 is located in an intronic region. Whereas the recognition of functional variants in the coding region is relatively simple, detecting changes in the noncoding region is more challenging due to the lack of a clear connection between nucleotide differences and regulatory functions. Some introns have been shown to contain transcription factor binding sites $[53,54]$ and to influence gene expression by several known and unknown mechanisms such as intron-mediated enhancement $[55,56]$. In this study, we speculated that the two alleles of rs7116978 may change potential binding sites for different transcription factors. We have performed TFBS (transcription factor binding site) prediction analysis in silico to verify this hypothesis. The two alleles were predicted to modify transcription binding sites of several transcription factors, including transcription factor 4 (TCF4) and the human glucocorticoid receptor (GR) (Supplementary Figure S2). Several studies have shown the link between these transcription factors with vitamin D metabolism. Beildeck et al. found that 1,25(OH)2D3 induces the expression of TCF4 in several human cell lines via a VDR-dependent mechanism [57]. As per the proposed model, TCF4 upregulation would enhance the repression of $\beta$-catenin/TCF target genes. With regards to the human glucocorticoid receptor (GR), cross-sectional studies have shown that the chronic use of glucocorticoids is associated with low levels of 25(OH)D [58]. It appears that the glucocorticoids can upregulate the renal expression of CYP24A1 which in turn catabolizes $25(\mathrm{OH}) \mathrm{D}$ and $1,25(\mathrm{OH}) 2 \mathrm{D}$ to water-soluble inactive agents, thus mediating deficiency of vitamin $\mathrm{D}[58,59]$. Another link between the use of glucocorticoids and vitamin $\mathrm{D}$ is that $1,25(\mathrm{OH}) 2 \mathrm{D} / \mathrm{VDR}$ and glucocorticoids/glucocorticoid receptor (GR) intracellular signaling pathways cross-talk so that the increased levels of vitamin D may enhance the responsiveness of certain target cells to glucocorticoids [60-62]. Glucocorticoids with their cognate receptors translocate from the cell cytoplasm to the nucleus where they bind to glucocorticoid response element (GRE) to regulate gene transcription. As VDR and GR share some coactivators, VDR may promote individual gene 
transcription induced by GR; additionally, it has been reported that vitamin D may upregulate the binding of GR to GRE [60]. These may result in vitamin D enhancing the cellular responsiveness to glucocorticoids. Functional studies are warranted to understand the importance of rs7116978 polymorphism as a potential regulator of gene expression.

The vitamin D molecular pathway is a complex process that varies across individual genetic profiles and according to their health status. As this current topic is still in its infancy, additional studies are warranted to elucidate how genetic variation contributes to vitamin D supplementation outcomes.

\section{Conclusions}

In conclusion, the present interventional study shows novel data about the association of vitamin D-related SNPs with responsiveness to vitamin D supplementation in individuals of Arab ancestry. Our findings should be validated in additional studies employing a cohort of a bigger size.

Supplementary Materials: The following are available online at http://www.mdpi.com/2072-6643/12/9/2608/s1, Figure S1: D' pairwise linkage disequilibrium (LD) plots of analyzed polymorphisms in Chromosomes 4, 11, 20 and 12, Figure S2: Transcription Factor Binding Sites Prediction for intronic SNP rs7116978. Table S1: SNP IDs and related sequences.

Author Contributions: S.K. designed the study. S.K. and E.S. planned the study. E.S. received funding for the study. M.A. and E.S. recruited the study subjects and performed the biochemical analysis. S.T., V.M., M.A. and R.M. processed the samples and analyzed the data. M.G. contributed to the data analysis. All authors discussed the results. S.T. wrote the draft of the manuscript. P.S. revised the first draft of the manuscript. All authors have read and agreed to the published version of the manuscript.

Funding: This study was supported by funds from Qatar University, Grant ID: QUCP-CHS- 17\18-1.

Acknowledgments: We thank the QU study participants. We thank Mohammed Elanbari for his help with the statistical analyses.

Conflicts of Interest: The authors declare no conflict of interest or relationships relevant to the content of this paper. All authors take responsibility for all aspects of the reliability of the data presented and their discussed interpretation.

\section{Abbreviations}

$\begin{array}{ll}\text { ALT } & \text { Alanine Aminotransferase } \\ \text { AST } & \text { Aspartate Aminotransferase } \\ \text { BMI } & \text { Body Mass Index } \\ \text { BUN } & \text { Blood Urea Nitrogen } \\ \text { HWE } & \text { Hardy-Weinberg Equilibrium } \\ \text { IFC } & \text { Integrated Fluidic Circuit } \\ \text { IRB } & \text { Institutional Review Board } \\ \text { LD } & \text { Linkage Disequilibrium } \\ \text { LSP } & \text { Locus-Specific Primer } \\ \text { PCR } & \text { Polymerase Chain Reaction } \\ \text { SNP } & \text { Single-Nucleotide Polymorphism } \\ \text { STA } & \text { Specific Target Amplification } \\ \text { TFBS } & \text { Transcription Factor Binding Site } \\ \text { PCA } & \text { Principal Component Analysis } \\ \text { QU } & \text { Qatar University } \\ \text { WC } & \text { Waist Circumference } \\ \text { 1000G } & \text { 1000 Genomes }\end{array}$

\section{References}

1. Santoro, D.; Caccamo, D.; Lucisano, S.; Buemi, M.; Sebekova, K.; Teta, D.; De Nicola, L. Interplay of vitamin D, erythropoiesis, and the renin-angiotensin system. Biomed. Res. Int. 2015, 2015, 145828. [CrossRef] [PubMed]

2. Holick, M.F. The vitamin D deficiency pandemic: Approaches for diagnosis, treatment and prevention. Rev. Endocr. Metab. Disord. 2017, 18, 153-165. [CrossRef] [PubMed] 
3. DeLuca, H.F. Vitamin D: Historical overview. Vitam. Horm. 2016, 100, 1-20. [CrossRef] [PubMed]

4. Jones, G.; Strugnell, S.A.; DeLuca, H.F. Current understanding of the molecular actions of vitamin D. Physiol. Rev. 1998, 78, 1193-1231. [CrossRef] [PubMed]

5. Elkum, N.; Alkayal, F.; Noronha, F.; Ali, M.M.; Melhem, M.; Al-Arouj, M.; Bennakhi, A.; Behbehani, K.; Alsmadi, O.; Abubaker, J. Vitamin D insufficiency in Arabs and South Asians positively associates with polymorphisms in GC and CYP2R1 genes. PLoS ONE 2014, 9, e113102. [CrossRef] [PubMed]

6. Holick, M.F. Vitamin D deficiency. N. Engl. J. Med. 2007, 357, 266-281. [CrossRef]

7. Erol, M.; Yigit, O.; Kucuk, S.H.; Bostan Gayret, O. Vitamin D deficiency in children and adolescents in Bagcilar, Istanbul. J. Clin. Res. Pediatr. Endocrinol. 2015, 7, 134-139. [CrossRef]

8. Djennane, M.; Lebbah, S.; Roux, C.; Djoudi, H.; Cavalier, E.; Souberbielle, J.C. Vitamin D status of schoolchildren in Northern Algeria, seasonal variations and determinants of vitamin D deficiency. Osteoporos. Int. 2014, 25, 1493-1502. [CrossRef]

9. Flores, M.; Macias, N.; Lozada, A.; Sanchez, L.M.; Diaz, E.; Barquera, S. Serum 25-hydroxyvitamin D levels among Mexican children ages 2 y to 12 y: A national survey. Nutrition 2013, 29, 802-804. [CrossRef]

10. Vierucci, F.; Del Pistoia, M.; Fanos, M.; Gori, M.; Carlone, G.; Erba, P.; Massimetti, G.; Federico, G.; Saggese, G. Vitamin D status and predictors of hypovitaminosis D in Italian children and adolescents: A cross-sectional study. Eur. J. Pediatr. 2013, 172,1607-1617. [CrossRef]

11. Singh, P.; Kumar, M.; Al Khodor, S. Vitamin D deficiency in the gulf cooperation council: Exploring the triad of genetic predisposition, the gut microbiome and the immune system. Front. Immunol. 2019, 10, 1042. [CrossRef] [PubMed]

12. Maalouf, G.; Gannage-Yared, M.H.; Ezzedine, J.; Larijani, B.; Badawi, S.; Rached, A.; Zakroui, L.; Masri, B.; Azar, E.; Saba, E.; et al. Middle East and North Africa consensus on osteoporosis. J. Musculoskelet. Neuronal. Interact. 2007, 7, 131-143. [PubMed]

13. Chakhtoura, M.; Rahme, M.; Chamoun, N.; El-Hajj Fuleihan, G. Vitamin D in the Middle East and North Africa. Bone Rep. 2018, 8, 135-146. [CrossRef] [PubMed]

14. Masoud, M.S.; Alokail, M.S.; Yakout, S.M.; Khattak, M.N.K.; AlRehaili, M.M.; Wani, K.; Al-Daghri, N.M. Vitamin D supplementation modestly reduces serum iron indices of healthy Arab Adolescents. Nutrients 2018, 10, 1870. [CrossRef]

15. Bassil, D.; Rahme, M.; Hoteit, M.; Fuleihan Gel, H. Hypovitaminosis D in the Middle East and North Africa: Prevalence, risk factors and impact on outcomes. Dermatoendocrinology 2013, 5, 274-298. [CrossRef]

16. Deluca, H.F. History of the discovery of vitamin D and its active metabolites. Bonekey. Rep. 2014, 3, 479. [CrossRef]

17. Dusso, A.S.; Brown, A.J.; Slatopolsky, E. Vitamin D. Am. J. Physiol. Renal. Physiol. 2005, 289 , F8-F28. [CrossRef]

18. Berlanga-Taylor, A.J.; Plant, K.; Dahl, A.; Lau, E.; Hill, M.; Sims, D.; Heger, A.; Emberson, J.; Armitage, J.; Clarke, R.; et al. Genomic response to vitamin d supplementation in the setting of a randomized, placebo-controlled trial. EBioMedicine 2018, 31, 133-142. [CrossRef]

19. Al-Daghri, N.M.; Yakout, S.M.; Wani, K.; Khattak, M.N.K.; Garbis, S.D.; Chrousos, G.P.; Al-Attas, O.S.; Alokail, M.S. IGF and IGFBP as an index for discrimination between vitamin D supplementation responders and nonresponders in overweight Saudi subjects. Medicine (Baltim.) 2018, 97, e0702. [CrossRef]

20. Kurylowicz, A.; Ramos-Lopez, E.; Bednarczuk, T.; Badenhoop, K. Vitamin D-binding protein (DBP) gene polymorphism is associated with Graves' disease and the vitamin D status in a Polish population study. Exp. Clin. Endocrinol. Diabetes 2006, 114, 329-335. [CrossRef]

21. Ramos-Lopez, E.; Bruck, P.; Jansen, T.; Herwig, J.; Badenhoop, K. CYP2R1 (vitamin D 25-hydroxylase) gene is associated with susceptibility to type 1 diabetes and vitamin D levels in Germans. Diabetes Metab. Res. Rev. 2007, 23, 631-636. [CrossRef] [PubMed]

22. Wang, T.J.; Zhang, F.; Richards, J.B.; Kestenbaum, B.; van Meurs, J.B.; Berry, D.; Kiel, D.P.; Streeten, E.A.; Ohlsson, C.; Koller, D.L.; et al. Common genetic determinants of vitamin D insufficiency: A genome-wide association study. Lancet 2010, 376, 180-188. [CrossRef]

23. Arabi, A.; Khoueiry-Zgheib, N.; Awada, Z.; Mahfouz, R.; Al-Shaar, L.; Hoteit, M.; Rahme, M.; Baddoura, R.; Halabi, G.; Singh, R.; et al. CYP2R1 polymorphisms are important modulators of circulating 25-hydroxyvitamin D levels in elderly females with vitamin insufficiency, but not of the response to vitamin D supplementation. Osteoporos. Int. 2017, 28, 279-290. [CrossRef] 
24. Zhang, Y.; Wang, X.; Liu, Y.; Qu, H.; Qu, S.; Wang, W.; Ren, L. The GC, CYP2R1 and DHCR7 genes are associated with vitamin D levels in northeastern Han Chinese children. Swiss Med. Wkly. 2012, 142, w13636. [CrossRef] [PubMed]

25. Bu, F.X.; Armas, L.; Lappe, J.; Zhou, Y.; Gao, G.; Wang, H.W.; Recker, R.; Zhao, L.J. Comprehensive association analysis of nine candidate genes with serum 25-hydroxy vitamin D levels among healthy Caucasian subjects. Hum. Genet. 2010, 128, 549-556. [CrossRef] [PubMed]

26. Ahn, J.; Yu, K.; Stolzenberg-Solomon, R.; Simon, K.C.; McCullough, M.L.; Gallicchio, L.; Jacobs, E.J.; Ascherio, A.; Helzlsouer, K.; Jacobs, K.B.; et al. Genome-wide association study of circulating vitamin D levels. Hum. Mol. Genet. 2010, 19, 2739-2745. [CrossRef]

27. Nissen, J.; Rasmussen, L.B.; Ravn-Haren, G.; Andersen, E.W.; Hansen, B.; Andersen, R.; Mejborn, H.; Madsen, K.H.; Vogel, U. Common variants in CYP2R1 and GC genes predict vitamin D concentrations in healthy Danish children and adults. PLoS ONE 2014, 9, e89907. [CrossRef]

28. Signorello, L.B.; Shi, J.; Cai, Q.; Zheng, W.; Williams, S.M.; Long, J.; Cohen, S.S.; Li, G.; Hollis, B.W.; Smith, J.R.; et al. Common variation in vitamin D pathway genes predicts circulating 25-hydroxyvitamin D Levels among African Americans. PLoS ONE 2011, 6, e28623. [CrossRef]

29. Wjst, M.; Altmuller, J.; Faus-Kessler, T.; Braig, C.; Bahnweg, M.; Andre, E. Asthma families show transmission disequilibrium of gene variants in the vitamin D metabolism and signalling pathway. Respir. Res. 2006, 7, 60. [CrossRef]

30. Hibler, E.A.; Klimentidis, Y.C.; Jurutka, P.W.; Kohler, L.N.; Lance, P.; Roe, D.J.; Thompson, P.A.; Jacobs, E.T. CYP24A1 and CYP27B1 polymorphisms, concentrations of vitamin D metabolites, and odds of colorectal adenoma recurrence. Nutr. Cancer 2015, 67, 1131-1141. [CrossRef]

31. Wu, X.; Cheng, J.; Yang, K. Vitamin D-related gene polymorphisms, plasma 25-hydroxy-vitamin D, Cigarette Smoke and Non-Small Cell Lung Cancer (NSCLC) risk. Int. J. Mol. Sci. 2016, 17, 1597. [CrossRef] [PubMed]

32. Engelman, C.D.; Fingerlin, T.E.; Langefeld, C.D.; Hicks, P.J.; Rich, S.S.; Wagenknecht, L.E.; Bowden, D.W.; Norris, J.M. Genetic and environmental determinants of 25-hydroxyvitamin D and 1,25-dihydroxyvitamin D levels in Hispanic and African Americans. J. Clin. Endocrinol. Metab. 2008, 93, 3381-3388. [CrossRef] [PubMed]

33. Hu, Z.; Tao, S.; Liu, H.; Pan, G.; Li, B.; Zhang, Z. The association between polymorphisms of vitamin D metabolic-related genes and vitamin D3 supplementation in type 2 diabetic patients. J. Diabetes Res. 2019, 2019, 8289741. [CrossRef]

34. Penna-Martinez, M.; Ramos-Lopez, E.; Stern, J.; Kahles, H.; Hinsch, N.; Hansmann, M.L.; Selkinski, I.; Grunwald, F.; Vorlander, C.; Bechstein, W.O.; et al. Impaired vitamin D activation and association with CYP24A1 haplotypes in differentiated thyroid carcinoma. Thyroid 2012, 22, 709-716. [CrossRef] [PubMed]

35. Orton, S.M.; Morris, A.P.; Herrera, B.M.; Ramagopalan, S.V.; Lincoln, M.R.; Chao, M.J.; Vieth, R.; Sadovnick, A.D.; Ebers, G.C. Evidence for genetic regulation of vitamin D status in twins with multiple sclerosis. Am. J. Clin. Nutr. 2008, 88, 441-447. [CrossRef]

36. Kuhn, T.; Kaaks, R.; Teucher, B.; Hirche, F.; Dierkes, J.; Weikert, C.; Katzke, V.; Boeing, H.; Stangl, G.I.; Buijsse, B. Plasma 25-hydroxyvitamin D and its genetic determinants in relation to incident myocardial infarction and stroke in the European prospective investigation into cancer and nutrition (EPIC)-Germany study. PLoS ONE 2013, 8, e69080. [CrossRef]

37. Miettinen, M.E.; Smart, M.C.; Kinnunen, L.; Keinanen-Kiukaanniemi, S.; Moilanen, L.; Puolijoki, H.; Saltevo, J.; Oksa, H.; Hitman, G.A.; Tuomilehto, J.; et al. The effect of age and gender on the genetic regulation of serum 25-hydroxyvitamin D-The FIN-D2D population-based study. J. Steroid. Biochem. Mol. Biol. 2018, 178, $229-233$. [CrossRef] [PubMed]

38. Rivera-Paredez, B.; Macias, N.; Martinez-Aguilar, M.M.; Hidalgo-Bravo, A.; Flores, M.; Quezada-Sanchez, A.D.; Denova-Gutierrez, E.; Cid, M.; Martinez-Hernandez, A.; Orozco, L.; et al. Association between Vitamin D deficiency and single nucleotide polymorphisms in the vitamin D receptor and GC genes and analysis of their distribution in Mexican postmenopausal women. Nutrients 2018, 10, 1175. [CrossRef]

39. McGrath, J.J.; Saha, S.; Burne, T.H.; Eyles, D.W. A systematic review of the association between common single nucleotide polymorphisms and 25-hydroxyvitamin D concentrations. J. Steroid Biochem. Mol. Biol. 2010, 121, 471-477. [CrossRef] 
40. Zhang, Z.; He, J.W.; Fu, W.Z.; Zhang, C.Q.; Zhang, Z.L. An analysis of the association between the vitamin D pathway and serum 25-hydroxyvitamin D levels in a healthy Chinese population. J. Bone Miner. Res. 2013, 28, 1784-1792. [CrossRef]

41. Holick, M.F.; Binkley, N.C.; Bischoff-Ferrari, H.A.; Gordon, C.M.; Hanley, D.A.; Heaney, R.P.; Murad, M.H.; Weaver, C.M.; Endocrine, S. Evaluation, treatment, and prevention of vitamin D deficiency: An endocrine society clinical practice guideline. J. Clin. Endocrinol. Metab. 2011, 96, 1911-1930. [CrossRef]

42. Al-Daghri, N.M.; Mohammed, A.K.; Bukhari, I.; Rikli, M.; Abdi, S.; Ansari, M.G.A.; Sabico, S.; Hussain, S.D.; Alenad, A.; Al-Saleh, Y.; et al. Efficacy of vitamin D supplementation according to vitamin D-binding protein polymorphisms. Nutrition 2019, 63-64, 148-154. [CrossRef]

43. Barrett, J.C.; Fry, B.; Maller, J.; Daly, M.J. Haploview: Analysis and visualization of LD and haplotype maps. Bioinformatics 2005, 21, 263-265. [CrossRef] [PubMed]

44. Hedrick, P.W. Gametic disequilibrium in cats: Epistasis is an unlikely cause. J. Hered. 1987, 78, 204. [CrossRef] [PubMed]

45. R Core Team. R: A Language and Environment for Statistical Computing; R Foundation for Statistical Computing: Vienna, Austria, 2013.

46. Jain, R.; von Hurst, P.R.; Stonehouse, W.; Love, D.R.; Higgins, C.M.; Coad, J. Association of vitamin D receptor gene polymorphisms with insulin resistance and response to vitamin D. Metabolism 2012, 61, 293-301. [CrossRef] [PubMed]

47. Iruzubieta, P.; Teran, A.; Crespo, J.; Fabrega, E. Vitamin D deficiency in chronic liver disease. World J. Hepatol. 2014, 6, 901-915. [CrossRef]

48. Franca Gois, P.H.; Wolley, M.; Ranganathan, D.; Seguro, A.C. Vitamin D deficiency in chronic kidney disease: Recent evidence and controversies. Int. J. Environ. Res. Public Health 2018, 15, 1773. [CrossRef]

49. Pike, J.W.; Meyer, M.B. The vitamin D receptor: New paradigms for the regulation of gene expression by 1,25-dihydroxyvitamin D3. Rheum. Dis. Clin. N. Am. 2012, 38, 13-27. [CrossRef]

50. Malik, S.; Fu, L.; Juras, D.J.; Karmali, M.; Wong, B.Y.; Gozdzik, A.; Cole, D.E. Common variants of the vitamin D binding protein gene and adverse health outcomes. Crit. Rev. Clin. Lab. Sci. 2013, 50, 1-22. [CrossRef]

51. Jones, G.; Prosser, D.E.; Kaufmann, M. Cytochrome P450-mediated metabolism of vitamin D. J. Lipid Res. 2014, 55, 13-31. [CrossRef]

52. Thacher, T.D.; Levine, M.A. CYP2R1 mutations causing vitamin D-deficiency rickets. J. Steroid Biochem. Mol. Biol. 2017, 173, 333-336. [CrossRef] [PubMed]

53. Wei, C.L.; Wu, Q.; Vega, V.B.; Chiu, K.P.; Ng, P.; Zhang, T.; Shahab, A.; Yong, H.C.; Fu, Y.; Weng, Z.; et al. A global map of $\mathrm{p} 53$ transcription-factor binding sites in the human genome. Cell 2006, 124, 207-219. [CrossRef] [PubMed]

54. Dahan, O.; Gingold, H.; Pilpel, Y. Regulatory mechanisms and networks couple the different phases of gene expression. Trends Genet. 2011, 27, 316-322. [CrossRef] [PubMed]

55. Gallegos, J.E.; Rose, A.B. An intron-derived motif strongly increases gene expression from transcribed sequences through a splicing independent mechanism in Arabidopsis Thaliana. Sci. Rep. 2019, 9, 13777. [CrossRef] [PubMed]

56. Mascarenhas, D.; Mettler, I.J.; Pierce, D.A.; Lowe, H.W. Intron-mediated enhancement of heterologous gene expression in maize. Plant Mol. Biol. 1990, 15, 913-920. [CrossRef] [PubMed]

57. Beildeck, M.E.; Islam, M.; Shah, S.; Welsh, J.; Byers, S.W. Control of TCF-4 expression by VDR and vitamin D in the mouse mammary gland and colorectal cancer cell lines. PLoS ONE 2009, 4, e7872. [CrossRef]

58. Skversky, A.L.; Kumar, J.; Abramowitz, M.K.; Kaskel, F.J.; Melamed, M.L. Association of glucocorticoid use and low 25-hydroxyvitamin D levels: Results from the National Health and Nutrition Examination Survey (NHANES): 2001-2006. J. Clin. Endocrinol. Metab. 2011, 96, 3838-3845. [CrossRef]

59. Hidalgo, A.A.; Trump, D.L.; Johnson, C.S. Glucocorticoid regulation of the vitamin D receptor. J. Steroid. Biochem. Mol. Biol. 2010, 121, 372-375. [CrossRef]

60. Zhang, Y.; Leung, D.Y.; Goleva, E. Anti-inflammatory and corticosteroid-enhancing actions of vitamin D in monocytes of patients with steroid-resistant and those with steroid-sensitive asthma. J. Allergy Clin. Immunol. 2014, 133, 1744-1752 e1741. [CrossRef] 
61. Obradovic, D.; Gronemeyer, H.; Lutz, B.; Rein, T. Crosstalk of vitamin D and glucocorticoids in hippocampal cells. J. Neurochem. 2006, 96, 500-509. [CrossRef]

62. Zhang, Y.; Leung, D.Y.; Goleva, E. Vitamin D enhances glucocorticoid action in human monocytes: Involvement of granulocyte-macrophage colony-stimulating factor and mediator complex subunit 14. J. Biol. Chem. 2013, 288, 14544-14553. [CrossRef] [PubMed]

(C) 2020 by the authors. Licensee MDPI, Basel, Switzerland. This article is an open access article distributed under the terms and conditions of the Creative Commons Attribution (CC BY) license (http://creativecommons.org/licenses/by/4.0/). 